University of Nebraska - Lincoln

DigitalCommons@University of Nebraska - Lincoln

Faculty Publications from Nebraska Center for

Materials and Nanoscience

Materials and Nanoscience, Nebraska Center for (NCMN)

February 2007

\title{
Granular layers on vibrating plates: Effective bending stiffness and particle-size effects
}

\author{
Wonmo Kang \\ University of Nebraska - Lincoln \\ Joseph A. Turner \\ University of Nebraska - Lincoln, jaturner@unl.edu \\ Florin Bobaru \\ University of Nebraska - Lincoln, fbobaru2@unl.edu \\ Liyong Yang \\ University of Nebraska - Lincoln, lyang4@unl.edu \\ Kitti Rattanidit \\ University of Nebraska - Lincoln
}

Follow this and additional works at: https://digitalcommons.unl.edu/cmrafacpub

Part of the Nanoscience and Nanotechnology Commons

\begin{abstract}
Kang, Wonmo; Turner, Joseph A.; Bobaru, Florin; Yang, Liyong; and Rattanidit, Kitti, "Granular layers on vibrating plates: Effective bending stiffness and particle-size effects" (2007). Faculty Publications from Nebraska Center for Materials and Nanoscience. 62.

https://digitalcommons.unl.edu/cmrafacpub/62

This Article is brought to you for free and open access by the Materials and Nanoscience, Nebraska Center for (NCMN) at DigitalCommons@University of Nebraska - Lincoln. It has been accepted for inclusion in Faculty Publications from Nebraska Center for Materials and Nanoscience by an authorized administrator of DigitalCommons@University of Nebraska - Lincoln.
\end{abstract}




\title{
Granular layers on vibrating plates: Effective bending stiffness and particle-size effects
}

\author{
Wonmo Kang, Joseph A. Turner, ${ }^{\text {a) }}$ Florin Bobaru, Liyong Yang, and Kitti Rattanadit \\ Department of Engineering Mechanics, University of Nebraska-Lincoln, W317.4 Nebraska Hall, \\ Lincoln, Nebraska 68588
}

(Received 2 February 2006; revised 3 November 2006; accepted 9 November 2006)

\begin{abstract}
Acoustic methods of land mine detection rely on the vibrations of the top plate of the mine in response to sound. For granular soil (e.g., sand), the particle size is expected to influence the mine response. This hypothesis is studied experimentally using a plate loaded with dry sand of various sizes from hundreds of microns to a few millimeters. For low values of sand mass, the plate resonance decreases with added mass and eventually reaches a minimum without particle size dependence. After the minimum, a frequency increase is observed with additional mass that includes a particle-size effect. Analytical nondissipative continuum models for granular media capture the observed particle-size dependence qualitatively but not quantitatively. In addition, a continuum-based finite element model (FEM) of a two-layer plate is used, with the sand layer replaced by an equivalent elastic layer for evaluation of the effective properties of the layer. Given a thickness of sand layer and corresponding experimental resonance, an inverse FEM problem is solved iteratively to give the effective Young's modulus and bending stiffness that matches the experimental frequency. It is shown that a continuum elastic model must employ a thickness-dependent elastic modulus in order to match experimental values. (C) 2007 Acoustical Society of America. [DOI: 10.1121/1.2404635]
\end{abstract}

PACS number(s): 43.40.At, 43.20.Tb, 43.40.Dx [JGM]

Pages: 888-896

\section{INTRODUCTION}

Interest in acoustic/seismic methods of detecting nonmetallic landmines has grown in recent years. Both linear ${ }^{1-4}$ and nonlinear $^{5-8}$ detection methods have shown good success in laboratory and field tests. These methods rely on the vibration response of the top plate of the mine under excitation from an acoustic wave. This response is complex due to the interaction of the plate with the soil which lies above the mine. Thus, a clear understanding of the response of a platesoil system is important for increasing the probability of detection and for reducing false alarms. The influence of a soil layer on the frequency response of a plate was recently examined experimentally by Korman and Sabatier ${ }^{9}$ for loess soil and by Zagrai et al. ${ }^{10}$ for moist sand. In all cases, it has been observed that the resonance decreases as the layer thickness increases, reaches a minimum and then increases. Here, a similar experiment is used to study the response of a plate loaded with sand. The emphasis is on the influence of particle size on the plate response. To explore the relation between particle size and the resonance analytically, a model based on effective medium theory and Hertzian contact is derived.

In addition, models of two-layer elastic plates are explored with regard to the measured data. First, the analytical solution of a two-layer plate is used and it is shown that such a model consistently overpredicts the observed resonant frequency. Next, a numerical model of the two-layer plate is used to describe the plate-sand behavior. The plate-sand sys-

\footnotetext{
a) Author to whom correspondence should be addressed; electronic mail: jaturner@unl.edu
}

tem examined experimentally is modeled as a thin-plate loaded by an equivalent elastic layer (EEL) which replaces the layer of sand. The nondissipative numerical model is then discretized by finite elements using shell elements for the thin metal plate and solid elements for the EEL. The equivalent elastic layer is defined as the layer that produces the shift/change in the first resonant frequency that matches the experimental measurements from the plate-sand system. This continuum-based model is selected due to its simplicity in evaluating effective elastic moduli for the granular layer. Its utility in evaluating the "bending stiffness" of a granular layer (a new concept defined here) is emphasized and the limits in capturing the interactions between the elastic plate and the real granular layer are examined.

In Sec. II, the experiments are described and results are presented. Then, the particle size effect observed experimentally is analyzed with an effective medium model for granular materials. In Sec. III, analytical and numerical models of the two-layer plate system are discussed. Also, the numerical method used to analyze the equivalent continuum threedimensional system is described. Finally, a summary and conclusions are presented in Sec. IV. The results are expected to provide insight into the importance of particle size effects for acoustic landmine detection.

\section{EXPERIMENTS AND EFFECTIVE MEDIUM MODELING}

The experiments are designed to provide insight into the relation between the particle size of sand layer and the resonant behavior of the plate. The experimental setup used here is shown schematically in Fig. 1 and is based on that of Korman and Sabatier. ${ }^{9}$ The plate-sand system is created us- 


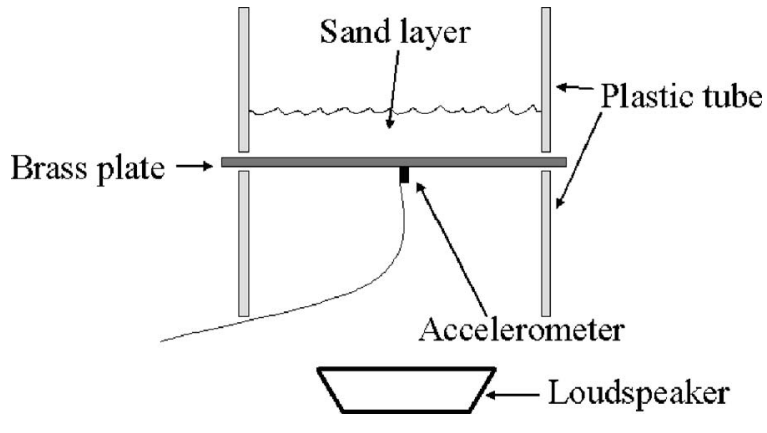

FIG. 1. Schematic of the cross section of the experimental setup. A thin brass plate is clamped between two plastic tubes and excited with a loudspeaker. The response is measured with an accelerometer mounted to the center of the plate.

ing a circular brass plate (mass $=26.4 \mathrm{~g}$, thickness $=0.25 \mathrm{~mm}$, radius $=50.8 \mathrm{~mm}$ ) clamped at the outer edge. Acoustic waves are generated by a loudspeaker positioned below the plate. A small input voltage of $50 \mathrm{mV}$ is used to ensure that the response remained in the linear regime for all measurements. The response of the plate is measured using an accelerometer placed at the center of the plate. The natural frequency corresponding to the system is determined using an HP35670A Dynamic Signal Analyzer. The first bending resonance for the brass plate alone is approximately $170 \mathrm{~Hz}$.

For this study, the influence of particle size is investigated. Thus, six different sizes of sand are used, all sieved from the same batch of dry sand. Additional measurements using the sand mixture are also made. The particle sizes and sample designations are shown in Table I. Note that all monodispersed samples have approximately the same effective density (the average density for S1-S6 is $1600 \pm 32.6 \mathrm{~kg} / \mathrm{m}^{3}$ ) indicating that the particles are packing essentially as spheres. However, the polydispersed sample (S-mix), from which the monodispersed samples were sieved, has an effective density $\sim 12 \%$ higher than the monodispersed average. The first bending resonance of the platesand system is measured using $10 \mathrm{~g}$ increments of sand, with a maximum layer thickness of $3.3 \mathrm{~cm}$.

\section{A. Experimental results}

The results of the experiments described above are presented in Fig. 2. The normalized resonant frequency, $\tilde{f}$ is defined as the frequency of the loaded plate divided by the frequency of the unloaded plate. Figure 2 shows the dependence of $\tilde{f}$ on the normalized mass of the sand layer. The overall trend of the resonance is similar to that observed for loess soil ${ }^{9}$ and moist sand. ${ }^{10}$ The resonance first decreases (region A), reaches a minimum and then increases (region

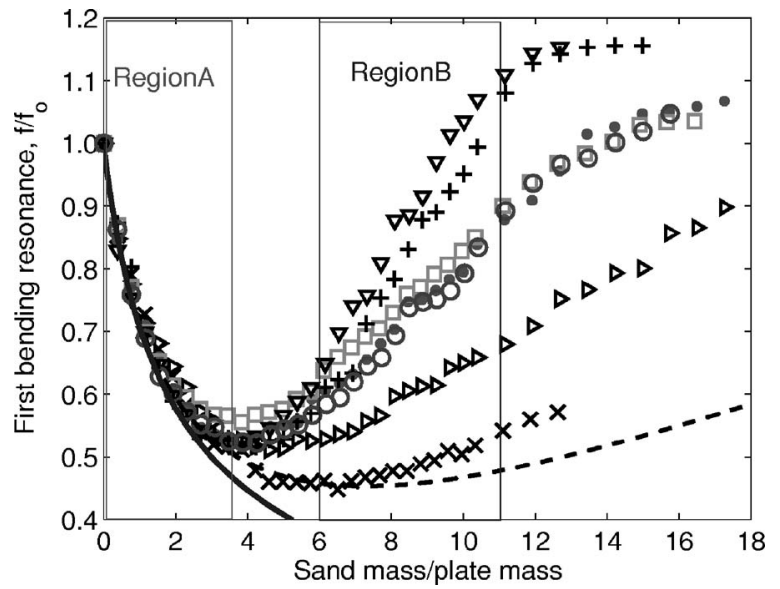

FIG. 2. Shift of the first bending resonance due to sand loading for seven samples of sand with various sizes (S1: $\times$, S2: $\triangleright$, S3: $\bullet$, S4: $\square$, S5: $\nabla$, S6: + , S-mix: $\bigcirc$, see Table I). The dashed line is based on Korman and Sabatier's data (Ref. 9), while the solid line is the behavior expected from mass loading alone as given by Eq. (3). In region A, there is no particle size effect observed. However, particle size becomes an important factor in region B.

B). It is clear from these results that the particle size is not relevant in Region A, but is important in region B. In addition, it is observed that larger particle sizes lead to higher resonant frequencies suggesting that the combined system is in some ways stiffer if larger particles are present. The universal nature of this response is discussed in the following. Another interesting result is also illustrated in Fig. 2. The curve denoted as S-mix is the result from the unsieved dry sand mixture (polydispersed). The size distribution of S-mix, shown in Table II, has an average particle size of $0.56 \mathrm{~mm}$. The response of the plate when loaded with this polydispersed mixture corresponds to the same curve $(0.3-0.6 \mathrm{~mm})$ as a monodispersed layer with the same average particle size although their effective densities are slightly different. This result suggests that the behavior of the system in region B depends only on the average particle size of the layer and is not as sensitive to the distribution of particle sizes in the layer.

Region A is a mass-dominated regime in which the combined system behaves as a plate loaded by added mass. Some understanding of the resonant frequency in region $\mathrm{A}$ is attained from the analytical solution of a clamped plate. The first bending frequency from classical thin plate theory is given by ${ }^{11}$

$$
\omega_{1}=\frac{\lambda_{1}^{2}}{\delta^{2}} \sqrt{\frac{D}{\rho}}=\frac{\lambda_{1}^{2}}{\delta^{2}} \sqrt{\frac{E h^{3}}{12 \rho\left(1-\nu^{2}\right)}},
$$

where $\lambda_{1}^{2}(=10.2)$ is the eigenvalue associated with the first bending mode of a clamped plate, $\delta$ is radius of the plate, $E$ is Young's modulus, $h$ is the plate thickness, $\rho$ is mass

TABLE I. Particle sizes and names of the samples of sand used for the experiments. All monodispersed samples (S1-S6) were sieved from the polydispersed mixture S-mix.

\begin{tabular}{|c|c|c|c|c|c|c|c|}
\hline Name & S1 & S2 & S3 & S4 & S5 & S6 & S-mix \\
\hline Size $(\mathrm{mm})$ & $<0.15$ & $0.15-0.3$ & $0.3-0.6$ & $0.6-1.18$ & $1.18-2.36$ & $2.36-4.75$ & Unsieved sand \\
\hline Density $\left(\mathrm{kg} / \mathrm{m}^{3}\right)$ & 1572 & 1574 & 1620 & 1660 & 1593 & 1570 & 1795 \\
\hline
\end{tabular}


TABLE II. Particle size distribution of samples S-mix, the polydispersed sample.

\begin{tabular}{crrrrrr}
\hline \hline Size & S1 & S2 & S3 & S4 & S5 & S6 \\
\hline Weight (g) & 17 & 209.3 & 344.4 & 156.7 & 41.4 & 5.1 \\
Ratio (\%) & 2.2 & 27.05 & 44.50 & 20.25 & 5.35 & 0.66 \\
\multicolumn{7}{c}{ Total weight of S-mix $=773.9 \mathrm{~g}$} \\
\hline \hline
\end{tabular}

density per unit area of the plate, and $\nu$ is Poisson's ratio. Equation (1) also serves to define the plate bending stiffness $D$. It can be assumed that the mass of sand is the dominant parameter in region A. Thus, the first bending resonance in region $\mathrm{A}, \omega_{A}$, can be written as

$$
\omega_{A}=\frac{\lambda^{2}}{\delta^{2}} \sqrt{\frac{D}{\rho_{\text {eff }}}}=\frac{\lambda^{2}}{\delta^{2}} \sqrt{\frac{D}{\rho_{p}(1+M)}},
$$

with an effective density defined as the average density of the plate-layer system $\rho_{\text {eff }}=\rho_{p}(1+M)$, where $M$ is mass ratio (mass of the sand normalized by the mass of the plate) and $\rho_{p}$ is the density of the brass plate per unit area $\left(=2.18 \mathrm{~kg} / \mathrm{m}^{2}\right)$. Thus, $\tilde{f}$ is given by

$$
\tilde{f}=\frac{f}{f_{0}}=\sqrt{\frac{1}{1+M}} .
$$

The solid line in Fig. 2 shows the trend expected from Eq. (3).

In region $\mathrm{B}$, the thickness of the sand layer is sufficient so that it behaves in many ways as a plate itself. In this region, the sand layer is dominant such that the brass plate has only a minor influence on the response of the plate-sand system. In this case, the first bending resonance of the plate in region $\mathrm{B}, \omega_{B}$, is given by

$$
\omega_{B}=\frac{\lambda^{2}}{\delta^{2}} \sqrt{\frac{E h_{\mathrm{eff}}^{2}}{12 \rho_{\mathrm{eff}}\left(1-\nu^{2}\right)}} \propto \sqrt{\frac{\left(h+M \rho_{p} / \rho_{s}\right)^{3}}{\rho_{p}(1+M)}},
$$

with the effective thickness of the plate-layer system given by $h_{\text {eff }}=\left(h+M \rho_{p} / \rho_{s}\right)$, where $\rho_{s}$ is the density of the sand layer. Equation (4) shows that when $M$ is sufficiently large, $\tilde{f}$ is expected to depend linearly on $M$. Thus, the trends observed in region $\mathrm{B}$ are also expected from the standpoint of basic plate vibrations. The dependence of the slope of $\tilde{f}$ on particle size in region $\mathrm{B}$ is discussed next, including expectations based on theoretical models of particle aggregates.

\section{B. Particle-size dependence}

As discussed earlier, the linear dependence of $\tilde{f}$ on $M$ is expected for the region of the measurements for which $M$ varies from 6 to 11. However, the slopes of these curves are clearly dependent on the average particle size of the layer as well as other parameters. In order to quantify this dependence, the slope of each curve $\sigma=\partial \tilde{f} / \partial M$ in region B is extracted. The slopes are calculated by fitting the entire $\tilde{f}-M$ curve using the function ${ }^{9}$

$$
\tilde{f}=\left[A /(M+1)+B M+C M^{2}\right]^{0.5},
$$

TABLE III. Slopes of the frequency curves for different particle sizes of sand.

\begin{tabular}{ccccccc}
\hline \hline Name & S1 & S2 & S3 & S4 & S5 & S6 \\
\hline$\sigma$ & 0.0387 & 0.0424 & 0.0728 & 0.0686 & 0.1153 & 0.1077 \\
\hline \hline
\end{tabular}

where $A, B$, and $C$ are fit coefficients. Then it is assumed that $\sigma=\sqrt{C}$ in the region of interest due to the relatively large value of $M$. The results are given in Table III. The dependence of $\sigma$ on particle radius $R$ is determined assuming a power-law relation. Thus, this dependence is given by

$$
\sigma(R)=\alpha R^{\beta},
$$

where $\alpha$ and $\beta$ are fit parameters obtained using the method of least squares. The results shown in Table III give a value for $\beta=0.305(\alpha=0.08)$. A plot of $\sigma$ as a function of $R$ is given in Fig. 3. The values of $\sigma$ and $R$ from the measurements are given in relation to Eq. (6).

The universality of this dependence is demonstrated using data from Korman and Sabatier. ${ }^{9}$ Their results are shown as the dashed line in Fig. 2 and are determined using Eq. (5) and their published coefficients $(A=1, B=0.0078$, and $C$ $=0.0004) .{ }^{9}$ The slope of their curve in region $\mathrm{B}$ is consistent with Eq. (6) for a particle size of $10.5 \mu \mathrm{m}$. The soil used by Korman and Sabatier (S-KS) was loess soil, a mixture of clay and silt. The result seems reasonable in that the particle size of clay is less then $2 \mu \mathrm{m}$ and that of silt is between 2 and $80 \mu \mathrm{m} .{ }^{12}$ The actual particle size of the S-KS soil was later confirmed as $10 \mu \mathrm{m} .{ }^{13}$ Thus, it appears that the powerlaw dependence proposed is sufficiently general for a large range of particle radii. However, the bending vibration response of a granular layer has not yet been considered from a theoretical standpoint.

The effective elastic response of an aggregate of particles has been of interest for over two decades. Although previous attempts to model such aggregates using effective medium theories have met with limited success, some progress has been made. For example Digby, ${ }^{14}$ Walton, ${ }^{15}$ and more recently Jenkins et al., ${ }^{16}$ have developed expressions for the effective elastic moduli of an aggregate of spherical

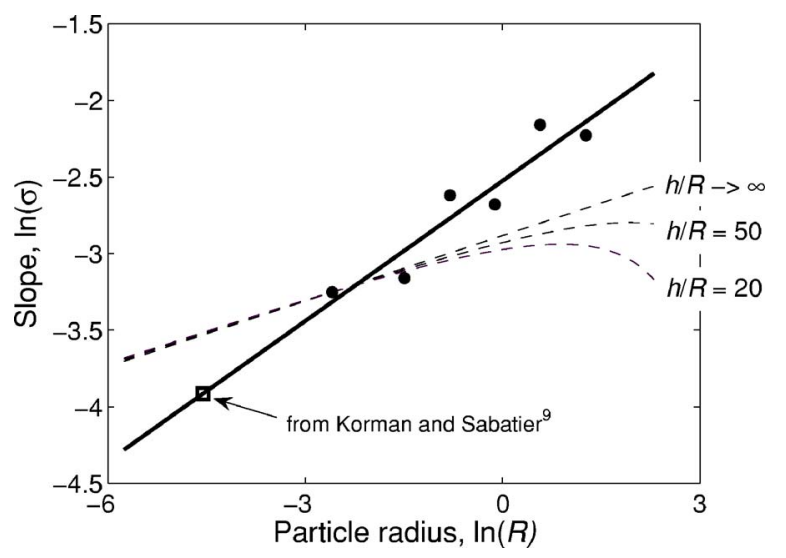

FIG. 3. Dependence of the slope of the resonance curves, $\sigma$, in region B on particle radius $R$. The experimental data from Table III are plotted as black circles. The power-law fit $\sigma=\alpha R^{\beta}$ is given by the solid line $(\alpha=0.08, \beta$ $=0.305$ ). This curve matches with data from Korman and Sabatier (Ref. 9). The dashed lines correspond to the analytical model for three values of $h / R$. 
particles under hydrostatic pressure. Here, their results are applied to the problem of interest such that the dependence of $\sigma$ on $R$ may be explored. As such, the resonant frequency of a circular plate of spherical particles with a clamped edge is assumed to have the form

$$
\omega_{B}=\frac{\lambda_{1}^{2}}{\delta^{2}} \sqrt{\frac{\bar{E} h_{s}^{3}}{12 \rho_{s}\left(1-\bar{\nu}^{2}\right)}},
$$

where $\bar{E}$ is the effective Young's modulus and $\bar{\nu}$ is Poisson's ratio of the effective plate with $h_{s}$ and $\rho_{s}$ as the thickness and density of the layer. Considering a random packing of identical elastic spherical particles of radius $R$, the effective Lamé's constants $\bar{\lambda}$ and $\bar{\mu}$ may be written ${ }^{14}$

$$
\begin{aligned}
& \bar{\lambda}=\frac{\mu C}{5 \pi R}\left[\frac{a}{1-\nu}-\frac{2 b}{2-\nu}\right], \\
& \bar{\mu}=\frac{\mu C}{5 \pi R}\left[\frac{a}{1-\nu}+\frac{3 b}{2-\nu}\right],
\end{aligned}
$$

where $\mu$ is shear modulus and $\nu$ is Poisson's ratio for the spheres. In addition, the packing has an average coordinate number $C=K \phi$, where $K$ is average number of contacts of each sphere and $\phi$ is the packing fraction. Each pair of grains is assumed to be initially bonded with a contact radius $b$. Under an applied hydrostatic pressure, the packing increases resulting in an increase in contact area with radius $a$, such that $a \geqslant b$. For the experiments described here, it is assumed that the contact of two particles without load is negligible, such that $b=0$. For the sand layer considered, the pressure from loading that results in the contact radius $a$ arises from the weight of the particles above the particle of interest. Thus, the effective Lamé constants may be rewritten in terms of the effective Young's modulus $\bar{E}$ and Poisson's ratio $\bar{\nu}$ as

$$
\begin{aligned}
& \bar{E}=\frac{\mu C}{\pi R} \frac{1}{2} \frac{a}{1-\nu}, \\
& \bar{\nu}=\frac{1}{4} .
\end{aligned}
$$

Under the assumption of Hertzian contact between two particles, the contact radius is given by ${ }^{17}$

$$
a=\left(\frac{3 p R}{4 E^{*}}\right)^{1 / 3},
$$

where $p$ is the applied normal force at the point of contact. As noted previously, ${ }^{18-20}$ no particle-size dependence results from this theory for a constant contact radius. To address this issue, it is assumed here that the contact force on a given particle contact is equal to the total weight of the particles above a given particle divided by the number of contacts over which the force is distributed. Thus, $p=p(z)=\rho_{s} g \pi(h$ $-z) /\left(3 z K \phi / 4 R^{3}\right)$, with $z$ the depth in the layer that varies from 0 to $h$. The average contact area is then given by

$$
\langle a\rangle=\left\langle\left(\frac{3 p(z) R}{4 E^{*}}\right)^{1 / 3}\right\rangle,
$$

where the ensemble average \langle\rangle , is an integral through the thickness of the layer. Thus, Eq. (11) becomes

$$
\langle a\rangle=\left(\frac{\rho_{s} g \pi}{E^{*} K \phi}\right)^{1 / 3} R^{4 / 3} \frac{1}{h} \int_{R}^{h}\left[\frac{h-z}{z}\right]^{1 / 3} d z
$$

where the reduced elastic modulus $E^{*}$ of the contact is given by $E^{*}=2 E /\left(1-\nu^{2}\right)$, with $E$ and $\nu$ as the Young's modulus and Poisson's ratio of the particles, respectively. Then the ratio of $\langle a\rangle / R$ can be expressed by

$$
\frac{\langle a\rangle}{R}=\left(\frac{\rho_{s} g \pi}{E^{*} K \phi}\right)^{1 / 3} R^{1 / 3} Q(R / h),
$$

where

$$
Q(R / h)=\int_{R / h}^{1}\left(\frac{1-\tilde{z}}{\tilde{z}}\right)^{1 / 3} d \widetilde{z} .
$$

Finally, the dependence of resonant frequency, Eq. (7), on $R$ is given by

$$
\omega_{B} \propto \sqrt{R^{1 / 3} Q(R / h)} .
$$

Thus, the final form given by Eq. (13) shows a dependence of the resonance on the size of particles in the layer. In order to put Eq. (13) in terms of the experimental results, the dependence of $\partial \tilde{f} / \partial M$ on $R$ is needed. This quantity is

$$
\sigma=\frac{\partial \tilde{f}}{\partial M} \propto \sqrt{R^{1 / 3} Q(R / h)},
$$

due primarily to the fact that the effective density of all particle sizes is equal. Example curves corresponding to Eq. (14) are shown in Fig. 3 for three values of $h / R$. It should be noted that for large layer thickness, $h / R \rightarrow \infty$ such that $Q(0)=2 \pi / 3 \sqrt{3}$. In this case, the dependence of $\sigma$ is $R^{1 / 6}$. The term $\sqrt{Q}$ reduces the value of $\sigma$ as shown. It is clear that Eq. (14) shows an increase in $\sigma$ with $R$, but that the exponent of the power-law dependence does not match the experimental result. Just as effective medium theories for granular systems have not been able to predict average moduli accurately, ${ }^{16}$ Eq. (14) does not accurately predict the size dependence of the frequency for the plate-sand system, although the general trend (increasing $\sigma$ for increasing $R$ ) is correct.

At this point it is unclear whether a more comprehensive effective medium approach, such as that developed by Jenkins et al., ${ }^{16}$ would yield results more closely related to the experiments. It is likely that the difference between the analytical estimates provided by these well-bonded models (two grains originally in contact remain in contact after an external load is applied) and the experimental results can be explained by the failure ${ }^{21}$ of the well-bonded model to approximate accurately the bending motion in the vibration of the granular system.

In Sec. III, the plate/sand-layer system is analyzed with the multilayer Kirchoff plate theory and a finite element model. The notion of the bending stiffness of the granular layer is of particular interest here. 


\section{TWO-LAYER PLATE MODELS}

It is clear from the experimental results shown above for the granular layer supported by a thin plate that a simple model is not sufficient to describe the observed behavior. Thus, additional aspects relevant for this system must be examined in order to identify the pertinent parameters that will allow predictive models to be developed. Toward that end, three different types of two-layer plate models are now explored. The first is an analytical model that describes the vibration behavior of a two-layer elastic plate. ${ }^{22}$ Such a model differs from the heuristic model presented previously ${ }^{10}$ for which the bending stiffnesses of the two layers were simply added. Unfortunately, the nature of the equation governing the vibrations of a multilayer plate does not support the approach in Ref. 10 from a physical standpoint. The second is a numerical approach using a finite element model (FEM). In this case, the upper layer is modeled using three-dimensional elements valid for thick plates. Finally, an inverse approach is presented for which the effective granular layer properties are extracted by matching the FEM results to the experimental results. This approach results in a thickness dependent modulus for the granular layer with corresponding bending stiffness (also thickness dependent).

\section{A. Analytical model}

The analytical solution for a two-layer elastic plate ${ }^{22}$ is now employed to model the system of interest. The top layer is considered as an EEL to study the dependence of the resonant frequency on the thickness of the granular layer. The majority of the derivation is not repeated here for brevity. The interested reader is referred to the original for more details. ${ }^{22}$ Both layers of the combined plate are modeled using Kirchhoff plate theory. The interface between the two layers is then assumed to be perfectly bonded such that there is continuity of displacement and stress. The boundary conditions of interest correspond to clamped conditions for both layers. Following standard vibration theory, harmonic solutions are sought and the spatial eigenvalue problem is derived. The solution of the eigenvalue problem results in the eigenfunctions (mode shapes) and allowable wave numbers (that are related to the natural frequencies) for the given geometry and boundary conditions. For the problem of interest here, the characteristic equation governing the natural frequencies is given by ${ }^{22}$

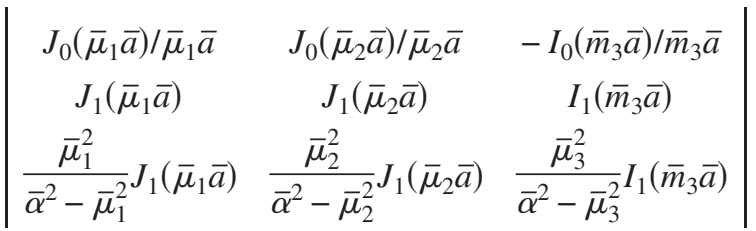

$$
\begin{aligned}
& =0 \text {, }
\end{aligned}
$$

where $J_{i}$ and $I_{i}$ are Bessel functions and modified Bessel functions of the first kind, respectively and $\bar{\alpha}^{2}=R_{0} \bar{\omega}^{2} / \bar{A}$. Here, $\bar{\omega}$ is the natural frequency of the two-layer plate and $\bar{a}$ is radius of the two-layer plate. Also, $R_{0}=\rho_{1} h_{1}+\rho_{2} h_{2}$ in terms of the mass densities $\left(\rho_{i}\right)$ of the plates and plate thicknesses $\left(h_{i}\right)$. The parameter

$$
\bar{A}=\frac{E_{1}}{1-\nu_{1}^{2}} h_{1}+\frac{E_{2}}{1-\nu_{2}^{2}} h_{2}
$$

is defined in terms of the moduli $\left(E_{i}\right)$ and Poisson's ratios $\left(\nu_{i}\right)$ of the plates. The dimensionless quantities $\bar{\mu}_{1} \bar{a}>0$, $\bar{\mu}_{2} \bar{a}>0$, and $\bar{\mu}_{3} \bar{a}=\bar{m}_{3} \bar{a} i$ in Eq. (15) are not independent, but are the roots of the following sixth-degree [third-degree in $\left.(\bar{\mu} \bar{a})^{2}\right]$ polynomial

$$
\begin{aligned}
& \left(1-\bar{B}^{2} / \bar{A} \bar{D}\right)(\bar{\mu} \bar{a})^{6}-\left(R_{0} \bar{\omega}^{2} / \bar{A}\right) \bar{a}^{2}(\bar{\mu} \bar{a})^{4} \\
& \left.\quad-\left(R_{0} \bar{\omega}^{2} / \bar{D}\right) \bar{a}^{4}(\bar{\mu} \bar{a})^{2}+\left(R_{0} \bar{\omega}^{2}\right)^{2} \bar{A} \bar{D}\right)=0 .
\end{aligned}
$$

In Eq. (16) $\bar{B}$ and $\bar{D}$ are defined as

$$
\begin{aligned}
& \bar{B}=-\frac{1}{2} \frac{E_{1}}{1-\nu_{1}^{2}} h_{1}^{2}+\frac{1}{2} \frac{E_{2}}{1-\nu_{2}^{2}} h_{2}^{2}, \\
& \bar{D}=\frac{1}{3} \frac{E_{1}}{1-\nu_{1}^{2}} h_{1}^{3}+\frac{1}{3} \frac{E_{2}}{1-\nu_{2}^{2}} h_{2}^{3},
\end{aligned}
$$

where $\rho_{1}$ and $\rho_{2}$ are mass densities of the plates, $h_{1}$ and $h_{2}$ are the thicknesses, $\nu_{1}$ and $\nu_{2}$ are the Poisson's ratios, $E_{1}$ and $E_{2}$ are the Young's moduli, and $\bar{\omega}$ is frequency of the twolayer plate. ${ }^{22}$ Equations (15) and (16) must be solved numerically for the necessary natural frequencies of the two-layer plate. From the brief description included here it is clear that the vibrations of a two-layer plate are much more complicated than a single-layer plate. For example, the solutions of Eq. (16) show that $\bar{\mu}_{1}, \bar{\mu}_{2}$, and $\bar{\mu}_{3}$ have nonlinear dependencies on the thickness ratio $h_{1} / h_{2}$ as do the natural frequencies. The mode shapes that result from the two-layer plate are also slightly different from those of a single-layer plate with the specific mode shapes dependent on the thickness ratio as well. These points are made to highlight the differences between the analytical two-layer plate theory ${ }^{22}$ and the model of Zagrai et al.: ${ }^{10}$ The two-layer plate theory has a more complicated characteristic equation and gives different mode shapes, wave numbers, and corresponding natural frequencies than those associated with a single-layer plate. An example result is shown in Fig. 4 for which the two-layer plate solution (brass plate with a top layer having $E$ $=3 \mathrm{MPa}$ ) is compared with Eq. (22) of Zagrai et al. Considerable differences are observed between the two results especially for most of the range of mass ratio.

Although the two-layer plate model discussed here has the appropriate qualitative behavior expected, it is not able to match the quantitative behavior of the experimental data. It is perhaps not too surprising since the Kirchhoff plate theory is valid for thin plates only (ratio of radius to thickness is at least 10-15). Thus, it is appropriate to explore a more applicable model that accounts for effects beyond the thin-plate theory.

\section{B. Finite element model}

The model discussed above is based on thin-plate theory. However, for much of the experimental data, the assumptions implicit in Eq. (4) are not expected to hold due to 


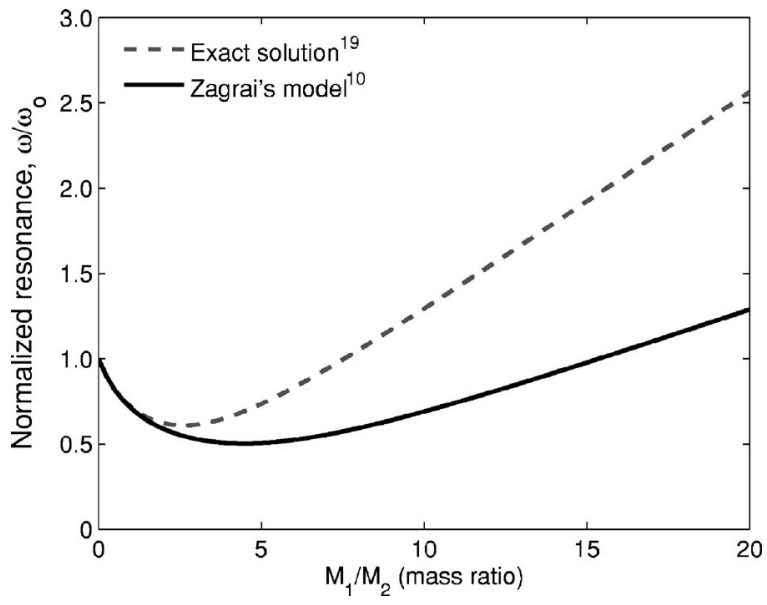

FIG. 4. Comparison between the exact solution (Ref. 22) for a two-layer plate and the heuristic model given in Ref. 10, Eq. (22).

thick-plate effects. In order to investigate the applicability of continuum models for this system further, the response of a thin plate loaded with an elastic layer is considered. The FEM is used to compute the first resonant mode of the twolayer system. The problem is axisymmetric such that axisymmetric elements can be used. Therefore, 57 SAX2 three-node axisymmetric shell elements and 115 CAX8R eight-noded biquadratic axisymmetric solid elements are used for the thin metal plate and the upper elastic layer, respectively. ${ }^{23}$ The selection of the number of shell elements is based on a convergence study performed to match the exact solution for the first resonant frequency of the thin plate to six digits. The mesh generator in ABAQUS automatically selects the number of solid elements corresponding to the number of shell elements and the input data. The number of elements in the thickness direction varies with the thickness of the sand layer. For example, 14 elements are employed in the thickness direction in the case of mass ratio of 5 . Due to the axisymmetry of the problem, the boundary condition corresponding to the center of the plate is chosen as rolling, while a clamped boundary condition is used for the plate outer edge. The boundary condition at the sand layer/tube interface (see Fig. 1) is expected to depend in some way on friction. The influence of this boundary condition on the numerical results is illustrated in Fig. 5, where results for both clamped and roller boundary conditions on the sand layer are compared. The results for a frictional boundary condition are expected to fall between these two extremes. The plots in Fig. 5 show that this boundary has only a minor influence on the predicted response for the regime of interest. Thus, a roller boundary condition is used for all simulations to follow. The condition along the interface between the plate and the EEL is rough friction (no slip occurs as relative horizontal motion of nodes in contact is prevented). Other interface conditions were examined as well but were found not to change results in any significant way.

The geometric and material properties of the plate are chosen to match the experimental system $\left(\delta=5.08 \times 10^{-2} \mathrm{~m}\right.$, $h=2.54 \times 10^{-4} \mathrm{~m}, \quad \rho=8575 \mathrm{~kg} / \mathrm{m}^{3}, \quad E=103 \mathrm{GPa}$, and $\nu$ $=0.34$ ). Analytical results for a two-layer plate ${ }^{22}$ and the FEM results show that changes in Poisson's ratio from 0.05

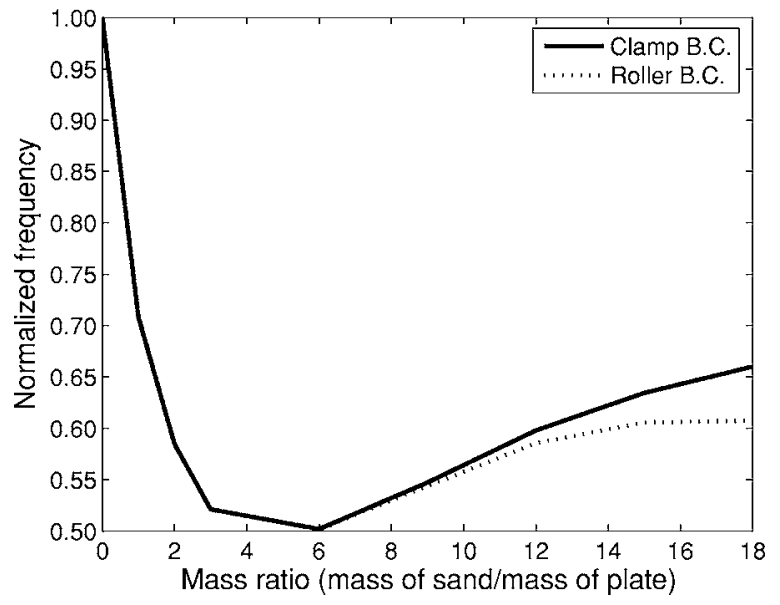

FIG. 5. Finite element results for the normalized first resonant frequency of the plate-EEL system for two types of boundary conditions (BC) applied at the interface between the tube walls and the EEL. The EEL replaces the granular layer of same thickness and density.

to 0.30 result in changes in the first resonant frequency smaller than $0.01 \%$. Thus, it is concluded that the first resonant frequency is insensitive to changes in $\nu$. Therefore, a Poisson's ratio of 0.25 is used for the EEL.

The ability of the numerical model to match the experimental results is first examined using a layer with the same density as that measured for the sand layer and with constant $E$. Numerical results are shown in Fig. 6 for $E=1,3,5 \mathrm{MPa}$, together with the analytical result for a two-layer elastic plate $(E=3 \mathrm{MPa})$ and three sets of experimental results (S1, S2, S5). In Fig. 6, the overall trends of the analytical two-layer plate theory and the three-dimensional FEM results are similar to the experiments: a decrease of the resonant frequency to a minimum then an increase with gradually decreasing rate as the thickness of the EEL increase. Note that the FEM result captures the leveling of the resonant frequency curve for large mass ratios. Such behavior is observed in the experiments but is impossible to obtain with the two-layer plate solution. However, neither the analytical solution for the two-layer plate nor the finite element models capture well the

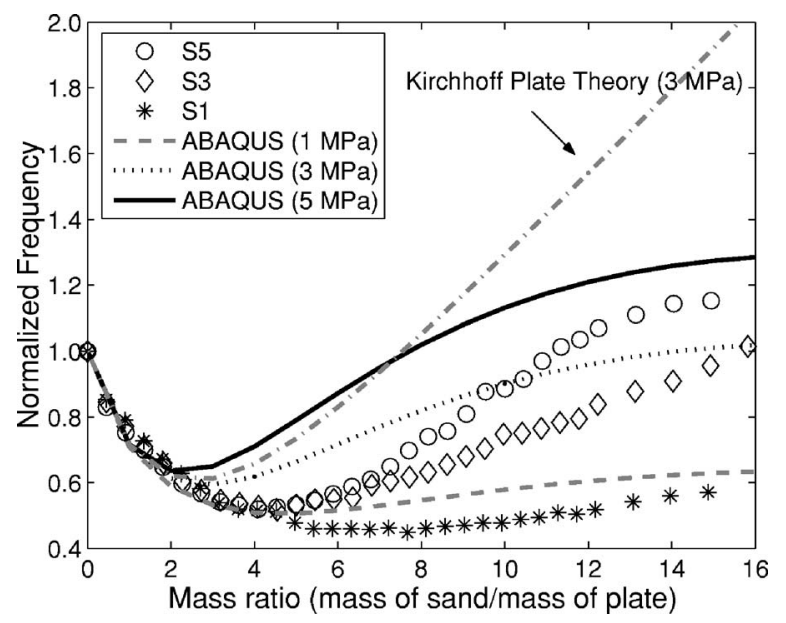

FIG. 6. Comparison of select experimental results with the two-layer plate model and the plate-EEL FEM model. The dash-dot line is obtained with the multilayer plate theory (Ref. 22) for $E=3 \mathrm{MPa}$ while the FEM results are obtained for equivalent elastic layers with $E=1,3$, and $5 \mathrm{MPa}$, respectively. 
location of the minimum and the slope in the region of increasing frequency. In fact, the results shown in Fig. 6 suggest that the elastic modulus of the layer depends on its thickness. In order to study this effect further, the FEM is used in an inverse analysis to determine the effective elastic properties of the sand layer.

\section{Thickness-dependent stiffness model}

The results thus far, based on two-layer plate theory and finite element model that includes three-dimensional effects of the layer, clearly show that neither can adequately model the measured frequency shift as a function of layer thickness. It seems that the granular layer has an effective bending stiffness that changes with thickness. In order to determine the properties of an EEL that produces the same resonant frequencies as in the experiments, the problem is formulated as an inverse problem. The objective is to find the appropriate Young's modulus from a given experimental resonance and thickness using a procedure based on the shooting method. ${ }^{24}$ For a given thickness of sand layer and the corresponding value of the first resonance from the experiment, the Young's modulus of an equivalent elastic layer of the same thickness is found as follows:

(1) Two arbitrary values of the Young's modulus $E_{0}$ and $E_{1}$ are chosen and the finite element model of the EEL-plate system is solved for both cases giving two values for the first resonant frequency, $\omega_{0}$ and $\omega_{1}$.

(2) Subsequent values for the Young's modulus are computed using

$$
E_{k+2}=E_{k}+\frac{\omega_{k}-\omega^{*}}{\omega_{k}-\omega_{k+1}}\left(E_{k+1}-E_{k}\right)
$$

for $k=0,1,2,3, \ldots$ where $\omega_{k}$ and $\omega_{k+1}$ are the resonant frequencies corresponding to $E_{k}$ and $E_{k+1}$, respectively, with $\omega^{*}$ denoting the experimental resonance.

(3) Iteration stops when the relative error between the experimental frequency and the frequency from the numerical model is less than a preset tolerance (here, $1 \%$ ).

This procedure is repeated for all experimental measurements (all particles sizes and all layer thicknesses).

The results from these computations in terms of Young's modulus of the EEL for all experiments are shown in Fig. 7 over the range of mass ratio from 4 to 16 . It should be noted that within the mass dominant regime for thin layers (region A in Fig. 2), the inverse problem of finding $E$ for the EEL from the experimental frequencies is ill-posed due to the insensitivity of the resonant frequencies to variations in the Young's values of the EEL. That is, small perturbations in the input frequencies can induce large changes in Young's moduli.

The values obtained for $E$ are in the range of several MPa for all sizes of sand examined, which is in the same range as those obtained by Yanagida et al. $^{25}$ for the "longitudinal elastic modulus" of sand using a different experiment when they analyzed properties of binary mixtures. The general trend observed is that layers of larger particles result in a larger effective $E$ of the EEL. The results also show that the

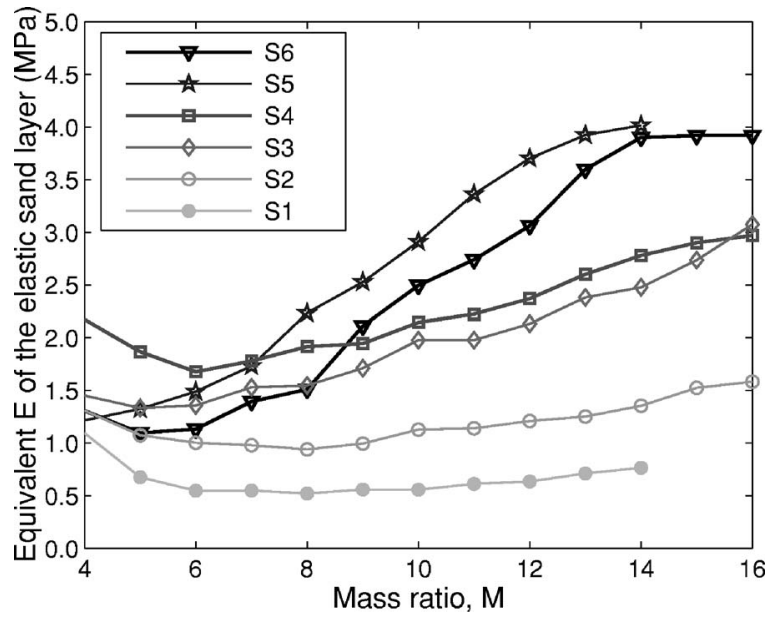

FIG. 7. Solution of the FEM-based inverse problem: computed values for the Young's modulus of an equivalent elastic layer that produces the same resonant frequency as the granular layer in the experiments (see Table I). Notice the general trend of increased elastic modulus with increase in particle size, as well as with the increase in thickness.

effective value of $E$ increases with the layer thickness for all particle sizes. It may be conjectured that this increase is due to an increase in the interparticle pressure from the layer as outlined in the model described in Sec. II B. It is also interesting to note that the values of $E$ shown in Fig. 6 are lower than might be expected based on wave speed measurements in sand $(103-260 \mathrm{~m} / \mathrm{s}),{ }^{17,26}$ which would result in a range of $E$ between 19 and $121 \mathrm{MPa}$.

In Fig. 8, the effective bending stiffness $D$ of the EEL, normalized by the bending stiffness of the plate, is plotted as a function of mass ratio. The general observations for $D$ are similar to those for $E$. However, when plotted in the context of the plate bending stiffness, the mass ratio necessary for the layer to become "stiffer" in bending than the metal plate is very clear. The results above the horizontal line $\left(D_{\mathrm{EEL}} / D_{p}=1\right)$ denote granular layers that dominate the vibration response of the plate/granular layer system. In terms of the particle size dependence, the results in Fig. 8 show that

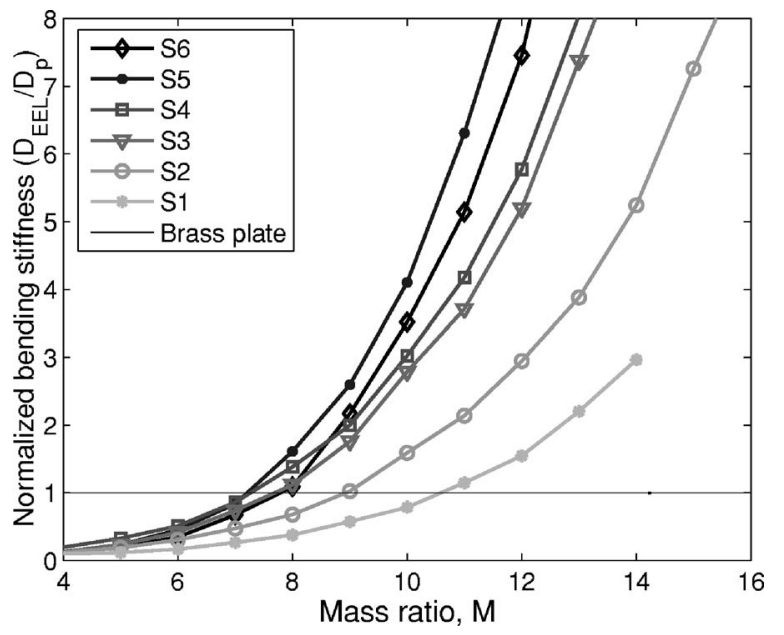

FIG. 8. Computed effective bending stiffness of the granular layer (see Table I) using the FEM-based inverse analysis for the equivalent elastic layer. Results are normalized by the bending stiffness of the metal plate. 
the EEL for larger particles achieves the same effective $D$ as the plate for layers that are thinner than the EEL for the smaller particles.

The inverse problem finite-element-based procedure described here provides a simple and effective means for computing the bending stiffness of a granular layer from experimental data. Such information is useful for the development of continuum models for granular media.

\section{SUMMARY}

In this article, it has been shown that the vibration response of a sand-loaded plate has two primary regimes. For layers with mass that are less than approximately five times the mass of the plate, the resonance exhibits a mass-loading behavior as predicted theoretically. In this regime the resonance decreases with a dependence $(1+M)^{-1 / 2}$ with $M$ as the mass ratio between the sand layer and plate. No dependence on particle size was observed. For higher values of $M$, the resonance reaches a minimum and then begins to increase with a clear dependence on average particle size: larger particles exhibit higher relative frequencies than smaller particles. The dependence on particle size is shown to behave according to a power law in particle radius. In addition, it was shown that the response to a polydispersed layer is equivalent to a monodispersed layer with the same average particle size. In other words, only the average particle size of a polydispersed layer appears to be important for this response. Attempts to understand this dependence based on effective moduli theories are qualitatively successful, but the value of the exponent in the power law does not match that of the experiments. The discrepancy between the observed exponent and the ones predicted by the well-bonded Hertzian models can perhaps be attributed to the failure of the model to include loss of contact and frictional dissipation in the bending motion induced by the vibration regime as well as other effects that are not clear at this time. This is the subject of future research.

In order to compute the bending stiffness of a granular layer a new methodology is proposed based on solving a sequence of inverse problems with the finite element method in which the metal-plate/granular layer system was replaced by an elastic shell/thick elastic layer numerical model. The nonlinear shooting method is used to obtain an elastic modulus for each thickness of the granular layer so that the equivalent elastic layer produces the same resonant frequency in the numerical model as that of the real system. It was shown that a single effective elastic modulus for the layers of a given particle size cannot be used to match the experimental results, but that a thickness-dependent modulus must be employed if the experimental data are to be matched.

These results show that layers of larger particles are "stiffer" than those made up of smaller particles for the same effective density. Modeling the dependence on particle size, however, remains to be elucidated since the effective medium theories based on well-bonded Hertzian models predict a value of the power law exponent that is half the size of that measured experimentally. At this point it is unclear if effec- tive medium theories can resolve this issue. In the future discrete models will be used to include rotational degrees of freedom and frictional dissipation: two of the missing ingredients that may be responsible for the above-mentioned discrepancy. Such models are clearly important for an improved understanding of the vibration response of an elastic structure that interacts with a granular material. Quantitative methods of land mine detection by acoustic means will necessarily require a clear grasp of such fundamental behavior.

\section{ACKNOWLEDGMENTS}

The research was sponsored by the U.S. Army Research Laboratory under the RMAC-RTP Cooperative Agreement No. W911NF-04-2-0011. The authors also thank M. S. Korman and J. M. Sabatier for helpful discussions and information regarding the experimental setup and their data from the soil/plate oscillator. Finally, an anonymous reviewer is thanked for referring us to Refs. 18-20.

${ }^{1}$ S. H. Lee, W. R. Scott, Jr., J. S. Martin, G. D. Larson, and G. S. McCall II, "Technical issues associated with the detection of buried land mines with high-frequency seismic waves," SPIE's Proceedings on Detection and Temediation Technologies for Mines and Minelike Targets VII, 2002, Vol. 4742, pp. 617-628.

${ }^{2}$ J. M. Sabatier and N. Xiang, "Laser-Doppler based acoustic-to-seismic detection of buried mines," SPIE's Proceedings on Detection and Remediation Technologies for Mines and Minelike Targets IV, 2003, Vol. 3710, pp. 215-222.

${ }^{3}$ W. R. Scott, Jr., S. H. Lee, G. D. Larson, J. S. Martin, and G. S. McCall II, "Use of high-frequency seismic waves for the detection of buried land mines," SPIE's Proceedings on Detection and Remediation Technologies for Mines and Minelike Targets VI, 2001, Vol. 4394, pp. 543-552.

${ }^{4}$ N. Xiang and J. M. Sabatier, "An experimental study on antipersonnel landmine detection using acoustic-to-seismic coupling," J. Acoust. Soc. Am. 113, 1333-1341 (2003).

${ }^{5}$ D. M. Donskoy, "Nonlinear vibro-acoustic technique for land mine detection," SPIE's Proceedings on Detection and Remediation Technologies for Mine and Minelike Targets III, 1998, Vol. 3392, pp. 211-217.

${ }^{6}$ D. Donskoy, A. Ekimov, N. Sedunov, and M. Tsionskiy, "Nonlinear seismo-acoustic land mine detection and discrimination," J. Acoust. Soc. Am. 111, 2705-2714 (2002).

${ }^{7}$ D. M. Donskoy and A. Ekimov, "Nonlinear vibrations of buried landmines," J. Acoust. Soc. Am. 117, 690-700 (2005).

${ }^{8}$ A. Zagrai, D. Donskoy, and A. Ekimov, "Resonance vibrations of buried landmines," SPIE's Proceedings on Detection and Remediation Technologies for Mines and Minelike Targets IX, 2004, Vol. 5415, pp. 21-29.

${ }^{9}$ M. S. Korman and J. M. Sabatier, "Nonlinear acoustic techniques for landmine detection," J. Acoust. Soc. Am. 116, 3354-3369 (2004).

${ }^{10}$ A. Zagrai, D. Donskoy, and A. Ekimov, "Structural vibrations of buried land mines," J. Acoust. Soc. Am. 118, 3619-3628 (2005).

${ }^{11}$ W. Leissa, Vibration of Plates (Acoustical Society of America, 1993).

${ }^{12}$ J. K. Mitchell, Fundamentals of Soil Behavior (Wiley, New York, 1976).

${ }^{13}$ M. S. Korman (private communication).

${ }^{14}$ P. J. Digby, "The effective elastic moduli of porous granular rocks," J. Appl. Mech. 48, 803-808 (1981).

${ }^{15} \mathrm{~K}$. Walton, "The effective elastic moduli of a random packing of spheres," J. Mech. Phys. Solids 35, 213-226 (1987).

${ }^{16}$ J. Jenkins, D. Johnson, L. LaRagione, and H. Makse, "Fluctuations and the effective moduli of an isotropic, random aggregates of identical, frictionless spheres," J. Mech. Phys. Solids 53, 197-225 (2005).

${ }^{17}$ K. L. Johnson, Contact Mechanics (Cambridge University Press, New York, 1985).

${ }^{18}$ M. Prasad and R. Meissner, "Attenuation mechanisms in sands: Laboratory versus theoretical (Biot) data," Geophysics 57, 710-719 (1992).

${ }^{19}$ R. Backrach, J. Dvorkin, and A. M. Nur, "Seismic velocities and Poisson's ratio of shallow unconsolidated sands," Geophysics 65, 559-564 (2000).

${ }^{20}$ M. Kimura, "Frame bulk modulus of porous granular marine sediments," J. Acoust. Soc. Am. 120, 699-710 (2006). 
${ }^{21}$ H. A. Makse, "Why effective medium theory fails in granular materials," Phys. Rev. Lett. 83, 5070-5073 (1999).

${ }^{22}$ Y. Stavsky and R. Loewy, "Vibration of isotropic composite circular plates," J. Acoust. Soc. Am. 49, 1542-1550 (1971).

${ }^{23}$ ABAQUS V.6.4/Standard.

${ }^{24}$ C. F. Gerald and P. O. Wheatley, Applied Numerical Analysis (Pearson Education, 2004).
${ }^{25}$ T. Yanagida, A. J. Matchett, B. N. Asmar, P. A. Langston, J. K. Walters, and J. M. Coulthard, "Dynamic response of well-mixed binary particulate systems subjected to low magnitude vibration," Adv. Powder Technol. 14, 589-604 (2003).

${ }^{26}$ M. L. Oelze, W. D. O’Brien, Jr., and R. G. Darmody, "Measurement of attenuation and speed of sound in soils," Soil Sci. Soc. Am. J. 66, 788796 (2002). 